\title{
STAPLELESS LAPAROSCOPIC ASSISTED RADICAL CYSTECTOMY WITH ILEAL NEOBLADDER IN A MALE AND WITH ILEAL LOOP IN A FEMALE: INITIAL REPORT FROM BRAZIL
}

\author{
SIDNEY C. ABREU, ROMULO A. SILVEIRA, JOAO B. CERQUEIRA, ROMMEL P. REGADAS, \\ LUCIO F. GONZAGA, GILVAN N. FONSECA
}

Sections of Urology, Federal University of Ceara and Federal University of Goias

\begin{abstract}
Introduction: Here, we report our initial experience with laparoscopic assisted radical cystectomy without the use of surgical staples.

Cases Report: A 70 year old male and a 55 year old female were diagnosed to have T2G3 transitional cell carcinoma of the bladder with negative metastatic work-out. Both patients were scheduled and agreed to a laparoscopic assisted radical cystectomy. In both cases, urinary diversion (orthotopic ileal Studer neobladder in the male and ileal conduit in the female) was performed extracorporeally following radical cystectomy. In both cases control of the bladder vascular pediclewas accomplished with a combination of metallic and hem-o-lock clips. The total surgical time was 6.5 hours in both cases. Estimated intra-operative blood loss was $500 \mathrm{cc}$ and $350 \mathrm{cc}$ respectively, however both patients required postoperative blood transfusions. No intraoperative complications occurred. In both cases, pathology revealed negative surgical margins.

Conclusions: Extracorporeal creation of urinary diversion decrease the overall operative time. Laparoscopic pelvic lymphadenectomy can be performed following the extended template. The use of surgical clips instead of vascular Endo-GIA titanium staples to control the bladder vascular pedicles is feasible and safe in selected patients, thus reducing intraoperative surgical costs. Considerable experience with laparoscopic radical prostatectomy is necessary before one attempts laparoscopic radical cystectomy.
\end{abstract}

Key words: bladder neoplasms; cystectomy; urinary diversion; lymphadenectomy; laparoscopy Int Braz J Urol. 2005; 31: 214-21

\section{INTRODUCTION}

The laparoscopic approach in urology is now an accepted option for kidney, adrenal, and prostate surgery. Naturally, this minimally invasive technique has progressed to the only remaining intra-abdominal urological organ left behind, the bladder. Although the first laparoscopic cystectomy for a retained bladder was performed over a decade ago (1), only recently, with the advancements in instrumentation design, and improvement of surgical techniques, laparoscopic radical cystectomy has regained interest (2).
Although feasible, some argue that radical cystectomy should not be performed laparoscopically, mainly because of issues related to a prolonged surgical time, the inability to perform an adequate lymph node dissection, and to a significant amount of disposables required, which elevates surgical costs. Recently, Basillote and colleagues have demonstrated that when cystectomy and pelvic lymph node dissection are performed laparoscopically and the reconstructive portion of the operation (ileal neobladder and ureteral re-implantation) is performed open surgically through a $15 \mathrm{~cm}$ Pfannensteil incision, 
the patient has the benefits of a minimally invasive approach, including decreased postoperative pain and faster recovery, without a significant increase in the operative time (3). Besides, the Cleveland Clinic group, highlighting the importance of extended pelvic lymphadenectomy in the management of bladder cancer, has demonstrated that during the laparoscopic procedure one should be able to perform the node dissection following the extended template, without compromising the established oncological open surgical principles (4). Furthermore, in an attempt to decrease surgical costs related to the use of disposable equipment, Abdel-Hakim et al. reported the use of the harmonic shears to control the lateral and posterior bladder pedicles, thus avoiding the use of 9 to 10 vascular loads of Endo-GIA stapler and reducing intraoperative costs (5).

Based on these promising results, we have decided to perform in our institution, a laparoscopic assisted radical cystectomy without the use of surgical staples. To the best of our knowledge, this is the first report of laparoscopic radical cystectomy with urinary reservoir in our country, placing Brazil among a selected group of 9 countries (14 different institutions) that have reported approximately 156 cases of laparoscopic radical cystectomy during the 2003 Annual Meeting of the American Urologic Association in Chicago, Illinois and the 2003 World Congress of Endourology in Montreal, Canada (2). Nonetheless, we do acknowledge that Mirandolino Mariano and his group have previously presented a video on laparoscopic cystectomy during the Iguaçu Falls meeting 2003 (6). However, these authors reported a salvage procedure without the construction of a urinary reservoir, only a bilateral cutaneous ureterostomy (6). Here, we describe our initial experience, technique and results with stapleless laparoscopic assisted radical cystectomy with ileal neobladder in a male and ileal loop in a female.

\section{SURGICAL TECHNIQUE}

\section{Laparoscopic Radical Cystoprostatectomy}

The patient was placed in the supine position with thighs abducted and arms adducted to his sides, the operative table was set in a $30-45^{\circ}$ Trendelenburg position and a Foley catheter was inserted in the bladder after the patient was prepped and draped. The surgeon was on the left side of the patient. The first assistant was on the right side of the patient and the second assistant was positioned next to the surgeon. A five-port transperitoneal approach was used. Following the creation of a $12-15 \mathrm{mmHg}$ of pressure $\mathrm{CO}^{2}$ pneumoperitoneum with a Verres needle, a primary $10 \mathrm{~mm}$ port was placed at the umbilicus for the $0^{\circ}$ laparoscope. Four secondary ports were placed under sight: a $5-12 \mathrm{~mm}$ port to the right and a $5 \mathrm{~mm}$ port to the left of the umbilicus, lateral to the rectus muscle, and two ports $(5 \mathrm{~mm})$ in the left and right lower quadrants, approximately 2 finger-breadths medial to the ipsilateral anterior superior iliac spines.

The posterior peritoneal fold was incised at the level of the common iliac vessel, the ureters were identified and widely mobilized bilaterally downwards close to the bladder wall. Adequate mobilization of the left ureter was assured to allow subsequent tension-free retroperitoneal transfer to the right side for the ureteroileal anastomosis. Subsequently, the peritoneum at the rectovesical pouch was incised in order to identify and the vas deferens and seminal vesicles, which were dissected towards the bladder base using the harmonic scalpel. Denonvilliers' fascia was incised with cold-cut scissors and dissection along the anterior rectal surface was followed distally towards the prostate apex. Both ureters were clipped close to the bladder and divided. The lateral and posterior bladder pedicles were then dissected and clip-ligated with a combination of metallic and hem-o-lock clips. Using the harmonic scalpel the neurovascular bundles were transected, leaving the prostate attached posteriorlly only by the rectourethralis muscles. The bladder was distended with $200 \mathrm{~mL}$ of saline and the parietal peritoneum lateral to the medial umbilical ligaments was incised and extended anteriorly onto the undersurface of the abdominal wall to include the entire urachus close to the umbilicus. The Retzius' space was entered and the bladder was mobilized keeping all the extraperitoneal perivesical fat attached to the bladder. The endopelvic fascia was incised bilaterally and the dorsal venous complex was suture ligated with 2-0 vicryl stitch on a CT-1 needle. This 
stitch was placed in a backhand manner from the right to the left side, distal to the prostate apex, between the dorsal vein complex and the urethra. In order to avoid inadvertent transgression of the urethra by the suture, the Foley catheter was replaced by an $18 \mathrm{Fr}$ metallic urethral sound, which was pushed down by the assistant, displacing the urethra posteriorly. In an attempt to achieve a safe ligation, two stitches were placed across the dorsal vein complex and were fastened secured (7). Using the J-hook electrocautery the dorsal vein complex was transected. At this point, cold Endoshears was used to transect the anterior and posterior urethral wall. The rectourethralis muscles were divided, thus completely freeing-up the surgical specimen, which was entrapped in a Lapsac for later extraction at the end of the procedure.

\section{Laparoscopic Radical Cystectomy in a Female}

The same port placement was employed with the patient in a low-lithotomy, $30-45^{\circ}$ Trendelenburg position. Since this patient had a prior pelvic surgery (ovariectomy), initial access was obtained with the Hasson technique and a $10 \mathrm{~mm}$ Blunt-tip balloon port was used at this site to prevent air leak. Following lise of intraperitoneal adhesions, the uterus was retracted anteriorly, and the initial peritoneotomy was made in order to identify the ureters, which were dissected down to the bladder wall, clip-ligated and transected. The peritoneum at the rectovesical culde-sac was incised and further dissection was performed to develop a plane anterior to the rectum. The ovary vascular pedicle, the uterine round and broad ligaments and vesical vascular pedicle were sequentially ligated with a combination of metallic and hem-o-lock clips and divided. Similarly to what was described above, the Retzius space was entered and the bladder was mobilized anteriorly. A sponge stick is inserted into the vagina to help identifying the vagina. Subsequently, the posterior vaginal wall was horizontally incised just distal to the uterine cervix with a J-hook electrocautery. This incision was then extended bilaterally towards the urethra, removing a strip of the anterior vaginal wall. At this point, the surgeon standing in between the patient's legs dissected the entire urethra circumferentially. The remaining attachments between the urethra and the anterior vaginal wall were cut and the intact surgical specimen (Figure-1) was extracted through the already open vaginal vault without the use of any bag. Pneumoperitoneum was re-established and maintained by keeping moister towels at the vaginal vault. Finally, the vaginal wall defect was closed laparoscopically with a 0 -vicryl running suture.

\section{Laparoscopic Extended Pelvic Lymph Node Dissection}

Bilateral extended pelvic lymphadenectomy was performed following radical cystectomy, including lymphatic tissue from the pubic bone distally to the bifurcation of the common iliac artery

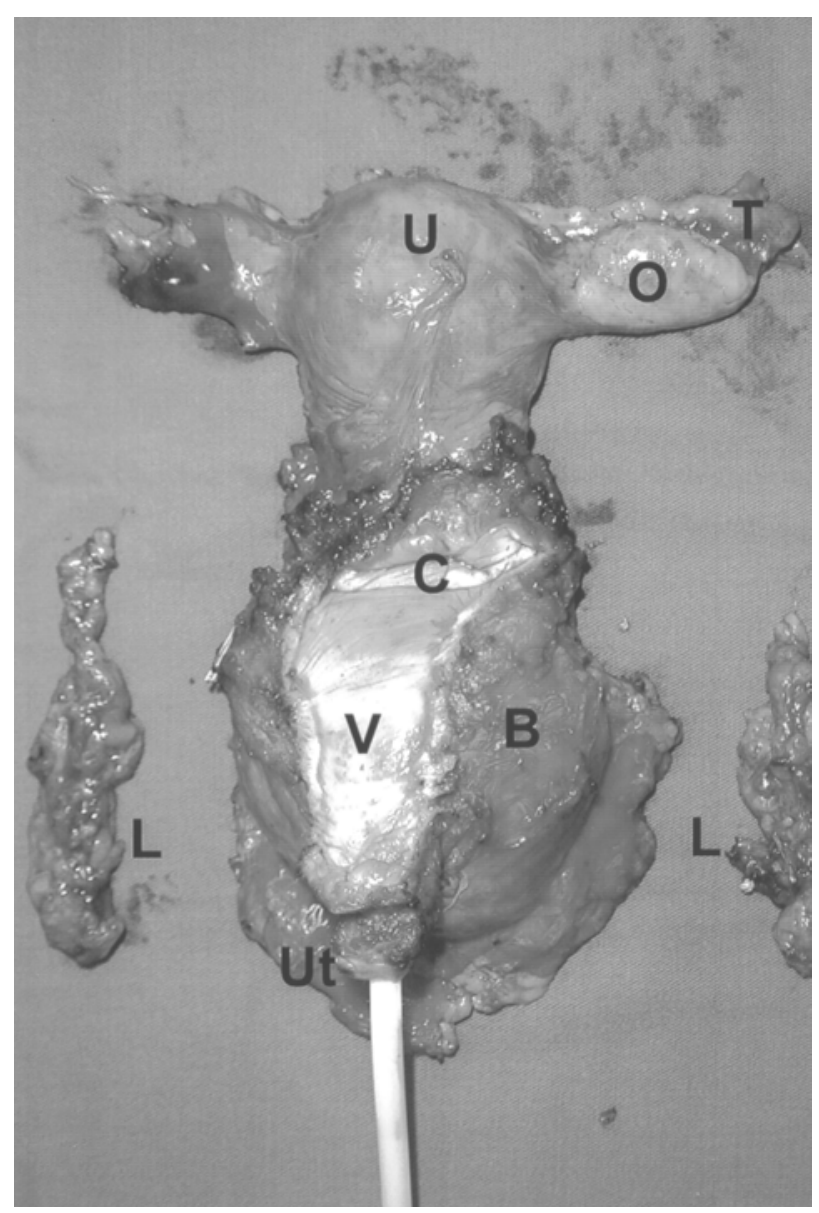

Figure 1 - Posterior view of the intact surgical specimen including, uterus $(U)$, uterine cervix $(C)$, tube $(T)$, ovary $(O)$, anterior vaginal wall $(V)$, bladder $(B)$, urethra $(U t)$, bilateral lymphatic package ( $L)$. 
proximally and from the genitofemoral nerve laterally and the obturator nerve inferiorly (4). The lateral border of dissection was developed along the genitofemoral nerve by dividing the fibro-areolar tissue and exposing the iliopsoas muscle. The lymphatic tissue packet was completely lifted en bloc off of the surface of the iliopsoas muscle and swept medially. The tissue anterior to the external iliac artery (up to the common iliac artery) and vein was then individually split longitudinally using $\mathrm{J}$ hook electrocautery, skeletonizing the 2 vessels circumferentially. The released packet was rolled medially posterior to the mobilized external iliac artery and vein, delivering it into the pelvis. Dissection along the medial aspect of the packet identified the obturator nerve. The entire specimen was placed in a Lapsac for further extraction.

\section{Urinary Diversion}

Orthotopic Ileal Neobladder - Following radical cystoprostatectomy and pelvic lymphadenectomy, a $12 \mathrm{~cm}$ Pfannensteil incision was made and the adnominal cavity was entered. The entrapped surgical specimen was then extracted. Bowel work and handling including: isolation of the distal ileal segment, creation of the Studer's neobladder, bilateral insertion of ureteral double J stents, refluxing bilateral ureteral re-implantation, and urethral-neobladder anastomosis over a 20F Foley catheter creation, was easily accomplished using standard open surgical techniques. Bilateral suction drains were left in the deep pelvis. No suprapubic cystostomy tube was employed.

Ileal Conduit - Following laparoscopic closure of the vaginal defect, the left ureter was delivered through the retroperitoneum to the right side. To facilitate a further identification, 2-0 vicryl stay sutures were placed at the tip of the ureters. Under laparoscopic control, the distal ileum was identified and grasped with a bowel clamp inserted though the right side $12 \mathrm{~mm}$ working port. Subsequently this port was removed, the port site incision was extended for $1.5 \mathrm{~cm}$, and the distal ileum was brought outside the abdominal cavity. A $15 \mathrm{~cm}$ ileal segment was isolated, its distal end was closed with a running suture and bowel continuity was restored. The mesenteric

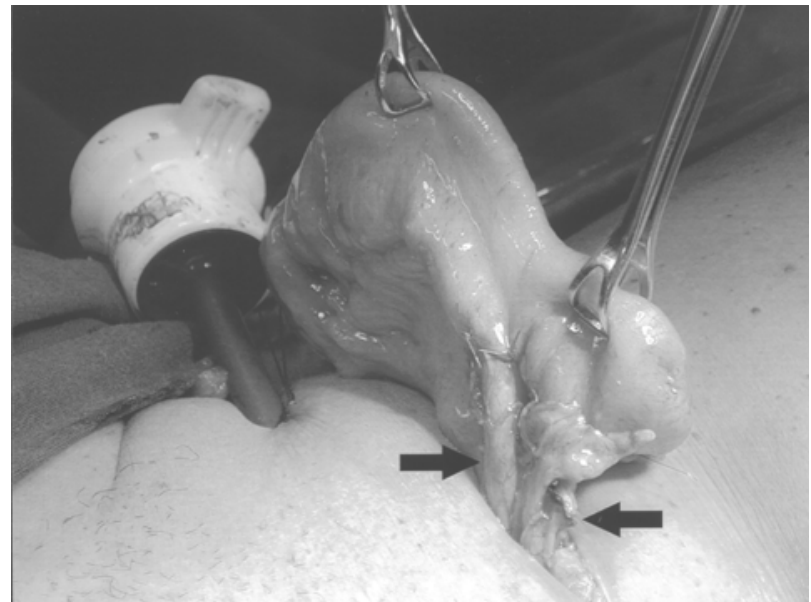

Figure 2 - Re-implanted ureters (arrows) and ileal loop exteriorized through the right side working port site.

window was closed and bowel was re-inserted inside the abdominal cavity, keeping the ileal loop outside. After gently occluding the right hand port site with 2 finger tips, the pneumoperitoneum was re-established and both ureters were identified and delivered outside the abdomen, through the right side working port site, under laparoscopic visualization. Standard bilateral stented ( 8 Fr NG tubes) ureteral-ileal loop anastomosis were performed open surgically (Figure2). The ileal loop along with the re-implanted ureters were re-inserted into the abdomen and an ileostomy was fashioned at the right hand working port site. A suction drain was left in the deep pelvis. The "neovagina" was packed with sterile gauzes.

\section{CASES REPORT}

\section{Case 1}

A 70 year old male, BMI of 24, ASA II, was diagnosed to have a T2G3 bladder transitional cell carcinoma. Metastatic workout with chest $\mathrm{x}$-ray and abdominal CT were negative. There were no sins of enlarged pelvic nodes or hydronephrosis. Patient was schedule and consent to a laparoscopic assisted radical cystoprostatectomy with orthotopic ileal neobladder.

Total surgical time was 6.5 hours, including 4.0 hours for radical cystectomy and pelvic lymphadenectomy and 2.5 hours for neobladder creation, intraoperative estimated blood loss was 


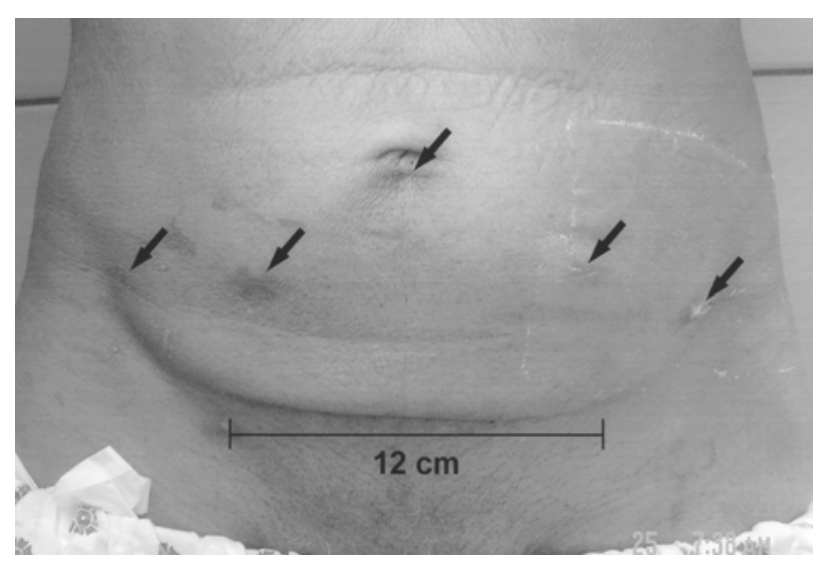

Figure 3 - Photography of port site incisions (arrows) and 12 cm Pfannenstiel incision took on postoperative day 21 when the patient was discharged.

$500 \mathrm{ml}$. Nonetheless, patients' postoperative hemoglobin dropped 4 points to $8.9 \mathrm{mg} / \mathrm{dL}$; hence he was given 2 units of concentrated red blood cells. No intraoperative complications occurred. However, the left side suction drain persisted with a high output of fluid $(700-1000 \mathrm{cc})$. A retrograde cystogram was then performed revealing only a small leakage at the neobladder-urethra anastomotic site. Measurement of creatinine levels in the drain fluid were similar to the serum. Biochemical analysis of the drain fluid showed a high concentration of triglycerides $(1015 \mathrm{ng} / \mathrm{dL})$ and cholesterol $(238 \mathrm{ng} / \mathrm{dL})$. The patient was treated successfully with dietary measures (high protein, low fat, medium chain triglyceride diet and salt restriction) for 3 weeks. At postoperative day 21, the Foley catheter along with the ureteral stents were removed and the patient was discharged home (Figure-3). Pathology revealed negative surgical margins.

\section{Case 2}

A 55 year old female, BMI 24, ASA II, heavy smoker, was diagnosed to have a T2G3 bladder transitional cell carcinoma. Metastatic workout with chest X-ray and abdominal CT were negative. There were no sins of enlarged pelvic nodes or hydronephrosis. Due to tumor extension to the bladder neck clearly seem during TURB, the decision was made to avoid an orthotopic pouch. The patient understood the risks and consented to a laparoscopic assisted radical cystectomy with total urethrectomy and ileal loop urinary diversion.

Total surgical time was 6.5 hours, including 5.0 hours for radical cystectomy and pelvic lymphadenectomy and 1.5 hour for ileal loop creation, estimated blood loss was $350 \mathrm{cc}$. No intra-operative complications occurred. Although intra-operative blood loss was low, the patient had a pre-operative hemoglobin of $10 \mathrm{mg} / \mathrm{dl}$ (after transfusion of 2 units of red blood cells); therefore we opted to transfuse one more unit of red blood cell base on a postoperative hemoglobin of $8.5 \mathrm{mg} / \mathrm{dL}$. Patient resumed fluids and ambulation on postoperative day 2 . The suction drain was removed on postoperative day 4 when the patient was discharged home.

\section{COMMENTS}

Laparoscopy is facing its last frontier in urological oncology, the surgical treatment of bladder cancer. As stated by Moinzadeh and Gill, this cutting edge procedure does offer advantages such as decreased blood loss, decreased postoperative pain, early return to full activity, and better cosmesis (2). Nonetheless, issues such as longer OR time, more expensive operation, and lack of long-term oncological data do represent a downside of this minimally invasive approach. Based on previous report in the literature, we have tried to address these cons related to laparoscopic radical cystectomy using the following strategy: a) In order to decrease the overall surgical time, we've performed the reconstructive part of the operation - urinary diversion extracorporeally. b) In order to comply with the oncological principles established in open surgery, we've followed an extended template for pelvic lymphadenectomy. c) In an attempt to reduce the surgical costs, we've avoided the use of laparoscopic surgical staples to control the bladder vascular pedicle.

Judging the laparoscopic procedure based on the operative time that is required to perform the same operation open surgically, we made the decision to fashion the urinary diversion intra or extracorporeally an important factor. In initial series where the reconstructive part of the operation (Mainz II sigmoid pouch, ileal conduit, Studer orthotopic ileal 
neobladder) performed completely intracorporeally, the total operation time varies from 7.4 hours to 10.5 hours (8-10). On the other hand, in our initial two cases, the urinary diversion (Studer neobladder or ileal loop) was performed extracorporeally, resulting in an overall surgical time of approximately 6.5 hours. Although this represents our initial experience with laparoscopic radical cystectomy, our group has familiarity with major laparoscopic surgery, including more than 25 laparoscopic radical prostatectomies successfully performed in the past year. This is in line with the majority of authors that do not recommend laparoscopic radical cystectomy before the surgeon has properly mastered the technique of laparoscopic radical prostatectomy (11). Whereas creation of the neobladder following laparoscopic cystectomy through a $12 \mathrm{~cm}$ Pfannensteil incision poses no significant difference to what is routinely performed during open surgery. The creation of the ileal loop and ureteral re-implantation through an extended 3 $\mathrm{cm}$ port site incision has inherent problems such as tissue orientation that could potentially lead to positional distortion. Moreover, it may be difficult or even impossible to extract the ileum and the ureters to the skin level in obese patients $(12,13)$.

There is a growing evidence in the literature supporting a surgical cure rate of $25 \%$ to $35 \%$ in patients with low volume lymph node disease. Moreover, recent studies suggest that the anatomical extent of lymphadenectomy is an important independent factor for the cure of bladder cancer (14-16). Following these well-established open surgical principles, Finelli and colleagues demonstrated that during the laparoscopic procedure one should be able to perform the node dissection following the extended template (4). Furthermore, these authors listed certain technical caveats such as, to perform lymphadenectomy after cystectomy so that tissue planes for laparoscopic cystectomy would not be compromised, to avoid iatrogenic trauma to any enlarged lymph node and to entrap the lymph node specimen immediately upon completing lymphadenectomy to minimize local and/or port site tissue contact (4). In our initial 2 cases, bilateral pelvic lymphadenectomy was performed without iatrogenic injury to the iliac vessels or to the obturator nerve $(17,18)$. However, in our first case a persistent postoperative chylous drainage occurred and was successfully managed with dietary measures (19). In order to avoid this problem in our second case, we've carefully clip-ligated any larger lymphatic channel prior to its transaction (4). Although the Cleveland Clinic group reported 2 cases of deep venous thrombosis (DVT) following laparoscopic extended pelvic lymphadenectomy (4). We did not experience any DVT in our 2two cases.

A higher intraoperative surgical cost, mainly related to the use of disposable equipment, represents a great obstacle to laparoscopic radical cystectomy gain acceptance, especially in developing countries (20). Traditionally, the control of the vascular pedicle of the bladder has been made employing serial Endo-GIA firing (9-10 loads of vascular $2.5 \mathrm{~mm}$ stapler) (5). As described by Abdel-Hakim and colleagues, the use of the harmonic shears could replace the Endo-GIA stapler for the control of the lateral and posterior pedicles of the bladder, thus reducing costs (5). In an attempt to further decrease the intraoperative costs, we've employed a combination of metallic and hem-o-lock clips to control the bladder vascular pedicle. Although it was safe in our initial experience, one should be bear in mind that we've performed these operations in selected thin patients, wherein the vascular pedicles of the bladder were not too thick, allowing precisely placement of surgical clips. It is also worth noticing that in the first case, following the removal of the $\mathrm{CO}^{2}$ pneumoperitoneum and cessation of its tamponade effect, a persistent oozing was observed coming from prostatic neurovascular bundles. We hypostasize that this continuous oozing persisted for a while after the procedure been responsible for the significant droop on hemoglobin levels postoperatively despite a reasonable $500 \mathrm{cc}$ intraoperative blood loss.

\section{CONCLUSIONS}

Extracorporeal creation of urinary diversion may help to decrease the overall operative time. However, a comparison to an intracorporeal diversion arm is required to truly evaluate this matter. Laparoscopic pelvic lymphadenectomy can be performed following the extended template. Use of surgical clips instead of vascular titanium staples to 
control the bladder vascular pedicle reduces intraoperative surgical costs. Stapleless laparoscopic assisted radical cystectomy with extended bilateral lymphadenectomy is feasible. Long-term oncological and functional follow-up is needed.

\section{REFERENCES}

1. Parra RO, Andrus CH, Jones JP, Boullier JA: Laparoscopic cystectomy: initial report on a new treatment for the retained bladder. J Urol. 1992; 148: 1140-4.

2. Moinzadeh A, Gill IS: Laparoscopic radical cystectomy with urinary diversion. Curr Opin Urol. 2004; 14: 837.

3. Basillote JB, Abdelshehid C, Ahlering TE, Shanberg AM: Laparoscopic assisted radical cystectomy with ileal neobladder: a comparison with the open approach. J Urol. 2004; 172: 489-93.

4. Finelli A, Gill IS, Desai MM, Moinzadeh A, MagiGalluzzi C, Kaouk JH: Laparoscopic extended pelvic lymphadenectomy for bladder cancer: technique and initial outcomes. J Urol. 2004; 172: 1809-12.

5. Abdel-Hakim AM, Bassiouny F, Abdel Azim MS, Rady I, Mohey T, Habib I, et al.: Laparoscopic radical cystectomy with orthotopic neobladder. J Endourol. 2002; 16: 377-81.

6. Mariano MB, Tefilli MV, Caldas P, IH Goldraich: Salvage laparoscopic cystoprostatectomy with cutaneous ureterostomy. Internat Braz J Urol. 2003; 29: (suppl. 29): Abst V017, 298.

7. Abreu SC, Gill IS: Pertinent issues related to laparoscopic radical prostatectomy. Int Braz J Urol. 2003; 29: 489-96.

8. Turk I, Deger S, Winkelmann B, Schonberger B, Loening SA, et al.: Laparoscopic radical cystectomy with continent urinary diversion (rectal sigmoid pouch) performed completely intracorporeally: the initial 5 cases. J Urol. 2001; 165: 1863-6.

9. Gill IS, Kaouk JH, Meraney AM, Desai MM, Ulchaker JC, Klein EA, et al.: Laparoscopic radical cystectomy and continent orthotopic ileal neobladder performed completely intracorporeally: the initial experience. J Urol. 2002; 168: 13-8.

10. Gill IS, Fergany A, Klein EA, Kaouk JH, Sung GT, Meraney AM, et al.: Laparoscopic radical cystoprostatectomy with ileal conduit performed completely intracorporeally: the initial 2 cases. Urology. 2000; 56: 26-9; discussion 29-30.
11. Mariano MB, Tefilli MV: Laparoscopic partial cystectomy in bladder cancer - initial experience. Int Braz J Urol. 2004; 30: 192-8.

12. Kozminski M, Partamian K: Case report of ileal loop conduit. J Endourol. 1992; 6: 147-50.

13. Sanchez de Badajoz E, Gallego Perales JL, Reche Rosado A, Gutierrez de la Cruz JM, Jimenez Garrido A: Laparoscopic cystectomy and ileal conduit: case report. J Endourol. 1995; 9: 59-62.

14. Vieweg J, Gschwend JE, Herr HW, Fair WR: Pelvic lymph node dissection can be curative in patients with node positive bladder cancer. J Urol. 1999; 161: 44954.

15. Stein JP, Lieskovsky G, Cote R, Groshen S, Feng AC, Boyd S, et al.: Radical cystectomy in the treatment of invasive bladder cancer: long-term results in 1,054 patients. J Clin Oncol. 2001; 19: 666-75.

16. Skinner DG: Management of invasive bladder cancer: a meticulous pelvic node dissection can make a difference. J Urol. 1982; 128: 34-6.

17. Hemal AK, Goel A: External iliac vein injury and its repair during laparoscopic radical cystectomy. JSLS. 2004; 8: 81-3.

18. Spaliviero M, Steinberg AP, Kaouk JH, Desai MM, Hammert WC, Gill IS: Laparoscopic injury and repair of obturator nerve during radical prostatectomy. Urology. 2004; 64: 1030.

19. Leibovitch I, Mor Y, Golomb J, Ramon J: The diagnosis and management of postoperative chylous ascites. J Urol. 2002; 167: 449-57.

20. Abreu SC, Fonseca G, Gadelha JB: Laparoscopic radical cystectomy with intracorporeally constructed Y-shaped ileal neobladder using non-absorbable titanium staples exclusively. Urology (in press), 2005.

Received: December 7, 2004

Accepted: March 19, 2005 


\section{EDITORIAL COMMENT}

The authors are to be congratulated for the pioneering in Brazil in performing laparoscopic assisted radical cystectomy and urinary diversion.

There are some papers published involving laparoscopic procedures over the bladder, but most of them experimental $(1,2)$. The majority of clinical papers focuses on the patients benefits of a minimally invasive procedure, but also, highlights the higher costs, the degree of difficulty and shorter follow-up when compared with open series (3-9). If one looks with criticism, would ask: Is there any real and profound advantage, regarding the patient, between the laparoscopic and open cystectomy? The answer probably would be no. Beyond the higher costs related to the laparoscopic approach, this technique still doesn't achieve the most important goal when cancer treatment is the subject: follow-up versus cure rate.

Even with the laparoscopic approach, the surgical morbidity is still high. The authors reported two cases and, both cases needed hemotransfusion $(100 \%)$. One case $(50 \%)$ remained in-hospital for 21 days, due to a chylous fistula, probably increasing too much the hospital cost. What can we say about a laparoscopic radical cystectomy and an open ileal neobladder through a $12 \mathrm{~cm}$ Pfannenstiel incision? Is that logical?

The authors courageously didn't use endoGIA staplers in order to decrease the costs. But, even using harmonic scalpel, an oozing was left behind, leading to a hemorrhage and consequently, hemotransfusion. The lateral bladder pedicles are very thick, maybe preventing only the use of clips, even Hem-O-Lok®.

It is very clear that the laparoscopic approach not only was, but still is the most important acquisition in the field of Urology of the last ten years. Nevertheless, everyone must bear in mind the limits. So, what and where is the surgical limit? We don't know yet, but we already know the human limit- the patient.
Many clinical and double-blinded research studies should be performed in order to achieve the answer.

So far, the open radical cystectomy and urinary diversion still is the "gold standard" treatment of muscle invasive bladder carcinoma.

\section{REFERENCES}

1. Paterson RF, Lifshitz DA, Beck SDW, Siqueira Jr TM, Cheng L, Lingeman JE, et al.: Multi-layered Small Intestinal Submucosa (MLSIS) is Inferior to Autologous Bowel for Laparoscopic Bladder Augmentation. J Urol. 2002; 168: 2253-7.

2. Siqueria Jr TM, Paterson RF, Kuo RL, Kaefer M, Cheng L, Shalhav AL: Laparoscopic Ileocytoplasty and Continent Ileovesicostomy in a Porcine Model. J Endourol. 2003; 17: 301-5.

3. Canes D, Triaca V, Tuerk I: Laparoscopic radical cystectomy with continent urinary diversion. Curr Urol Rep. 2005; 6: 109-17.

4. van Velthoven RF, Piechaud T: Laparoscopic radical cystectomy with ileal conduit diversion. Curr Urol Rep. 2005; 6: 93-100.

5. Simonato A, Gregoria A, Lissiani A: Laparoscopic radical cystectomy and urinary diversion: fad or future? BJU Int. 2004; 94:1399-400.

6. DeGer S, Peters R, Roigas J, Wille AH, Tuerk IA, Loening SA: Laparoscopic radical cystectomy with continent urinary diversion (rectosigmoid pouch) performed completely intracorporeally: an intermediate functional and oncologic analysis. Urology. 2004; 64: 935-9.

7. Hrouda D, Adeyoju AA, Gill IS: Laparoscopic radical cystectomy and urinary diversion: fad or future? BJU Int. 2004; 94: 501-5.

8. Basillote JB, Abdelshehid C, Ahlering TE, Shanberg AM: Laparoscopic assisted radical cystectomy with ileal neobladder: a comparison with the open approach. J Urol. 2004; 172: 489-93.

9. Hemal AK, Kumar R, Seth A, Gupta NP: Complications of laparoscopic radical cystectomy during the initial experience. Int J Urol. 2004; 11: 483-8.

Dr. Tibério M. Siqueira Jr. Getulio Vargas Hospital Recife, PE, Brazil 